\title{
BMJ Open Rate of adherence to urate-lowering therapy among patients with gout: a systematic review and meta-analysis
}

\author{
Rulan Yin, ${ }^{1,2}$ Lin Li, ${ }^{3}$ Guo Zhang, ${ }^{4}$ Yafei Cui, ${ }^{3}$ Lijuan Zhang, ${ }^{3}$ Qiuxiang Zhang, ${ }^{3}$ \\ Ting Fu, ${ }^{1}$ Haixia Cao, ${ }^{5}$ Liren $\mathrm{Li}^{3}{ }^{3}$ Zhifeng Gu${ }^{1,5}$
}

To cite: Yin R, Li L, Zhang G, et al. Rate of adherence to urate-lowering therapy among patients with gout: a systematic review and meta-analysis. BMJ Open 2018;8:e017542. doi:10.1136/ bmjopen-2017-017542

- Prepublication history and additional material for this paper are available online. To view these files, please visit the journal online (http://dx.doi. org/10.1136/bmjopen-2017017542).

RY, LL and GZ contributed equally.

Received 1 May 2017 Revised 28 September 2017 Accepted 2 0ctober 2017

Check for updates

${ }^{1}$ Research Center of Clinical Medicine, Affiliated Hospital of Nantong University, Nantong, China

${ }^{2}$ Emergency and critical care medicine, The First Affiliated Hospital of Soochow University, Suzhou, China

${ }^{3}$ School of Nursing, Nantong University, Nantong, China

${ }^{4}$ Department of Operating Room, The First Affiliated Hospital of Soochow University, Suzhou, China

${ }^{5}$ Department of Rheumatology, Affiliated Hospital of Nantong University, Nantong, China

Correspondence to

Liren Li; larry017@163.com and Dr Zhifeng Gu; guzf@ntu.edu.cn

\section{ABSTRACT}

Introduction Reported adherence to urate-lowering

therapy (ULT) in gout varies widely (17\%-83.5\%).

Variability may partly be due to different adherence measurement methods. This review aimed to quantify ULT adherence in adult patients with gout.

Methods This analysis examined studies in PubMed, Web of Science, CNKI Scholar and WanFang databases from inception to January 2017. Papers were selected by inclusion and exclusion criteria in the context. Randomeffect meta-analysis estimated adherence.

Results 22 studies were found by the inclusion criteria, which involved 137699 patients with gout. Four ways to define adherence were reported. Meta-analysis revealed that the overall adherence rate was $47 \%(95 \% \mathrm{Cl} 42 \%$ to $\left.52 \%, I^{2}=99.7 \%\right)$. Adherence rate to ULT was $42 \%(95 \% \mathrm{Cl}$ $37 \%$ to $\left.47 \%, l^{2}=99.8 \%\right)$ for prescription claims, $71 \%(95 \%$ Cl $63 \%$ to $79 \%$ ) for pill count, $66 \%$ (95\% Cl $50 \%$ to $81 \%$, $\left.\mathrm{I}^{2}=86.3 \%\right)$ for self-report and $63 \%(95 \% \mathrm{Cl} 42 \%$ to $83 \%$, $\left.\mathrm{I}^{2}=82.9 \%\right)$ for interview, respectively. The influential factor on adherence rate was country of origin.

Conclusions Among adult patients with gout, overall adherence rate to ULT was as low as $47 \%$, which suggested that clinicians should pay more attention to medication adherence in patients with gout to effectively improve adherence to ULT.

\section{INTRODUCTION}

Gout, which is characterised by the deposition of monosodium urate monohydrate in the synovial fluid and other tissues, is the most common cause of inflammatory arthritis worldwide. ${ }^{1}$ A treat-to-target serum urate (SU) strategy for patients with gout with an indication for urate-lowering therapy (ULT), such as allopurinol, febuxostat or probenecid, has been widely endorsed as a means of optimising clinical outcomes. ${ }^{2}$ Previous studies have reported that effective ULT reduce SU levels sufficiently to prevent further crystal formation and to dissolve existing urate crystals, thus eliminating the causative agent, making gout the only chronic arthritis that can be 'cured'. ${ }^{3-5}$ Therefore, lifelong ULT prescription, the key to successful long-term management of gout, ${ }^{6}$ is usually advised.

\section{Strengths and limitations of this study}

To the best of our knowledge, this was the first meta-analysis quantifying the overall adherence rate to urate-lowering therapy (ULT) in patients with gout.

- This systematic review was composed of 22 studies, with 137699 patients with gout.

- A substantial amount of heterogeneity among the studies remained unexplained by the variables examined.

- EMBASE database and Cochrane database library were not searched owing to lack of access.

- Several studies that referred to medications unspecified ULT were excluded, which could bias the findings.

But the prospect of lifelong therapy may contribute to very low adherence rate. ${ }^{7} \mathrm{~A}$ $\mathrm{WHO}$ report indicated that if patients with long-term therapies had poor adherence, the effectiveness of treatment may be impaired. ${ }^{8}$ Therefore, it is significant to understand the measurement and determinants of adherence in gout. However, reported ULT adherence rates in patients with gout vary between $10 \%$ and $46 \%$ in different studies. ${ }^{9}$ The vast interstudy difference may partly result from different adherence measurement methods, as well as definition of adherence. Our purpose was to establish pooled prevalence of adherence to ULT in patients with gout with regard to different measurement methods. This context assumed that measurement methods will affect the adherence rates obtained.

From what we know, this is the first attempt to estimate adherence rate to ULT in gout, for different adherence measurement methods. Variability of cut-points to define adherence is also explored across different studies.

\section{METHODS}

The meta-analysis was reported according to the recommendations of Preferred Reporting 


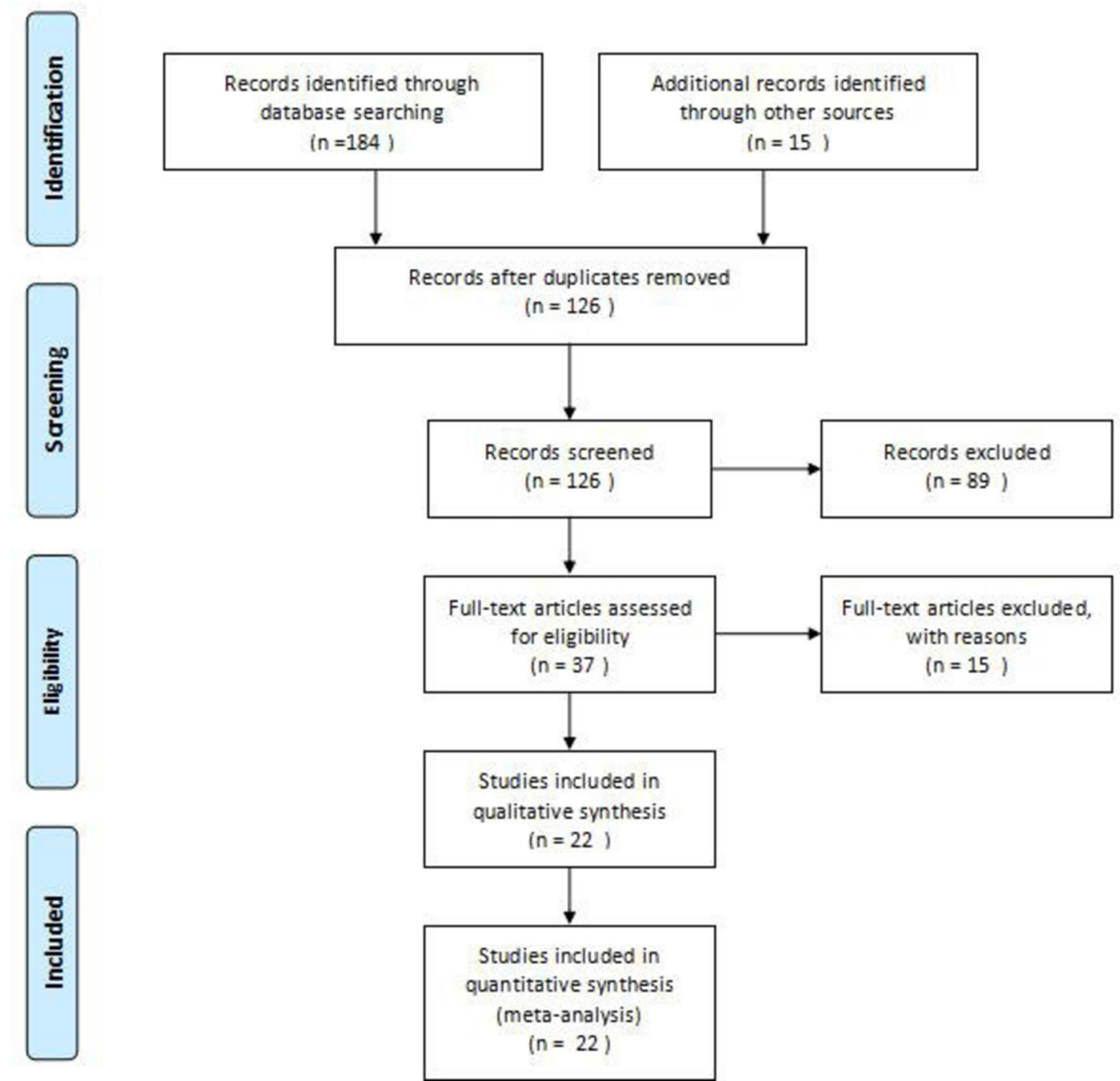

Figure 1 Flow chart illustrating the article search process. First, we obtained 184 records identified through database searching, and 15 additional records identified through other sources. Second, 126 records remained after duplicates were removed. Third, 89 studies were excluded after records screening. Then the remaining 37 studies were assessed for eligibility of which 15 studies were excluded. Finally, 22 studies were included in the quantitative synthesis (meta-analysis).

Items for Systemic Reviews and Meta-Analyses and the Meta-analysis of Observational Studies in Epidemiology as closely as possible. ${ }^{1011}$

\section{Search strategy}

The systematic review examined the English-language databases of PubMed and Web of Science, and Chinese databases of the CNKI Scholar and WanFang (from inception to January 2017) to identify related studies; we also searched references that were listed in the studies. Reviews were used to identify relevant articles and to proof the search strategy. Case reports, letters and editorials were not included as primary data.

Different search strategies were combined, as follows. For the English-language databases, search details were (adherence [All Fields] OR ('patient compliance' [MeSH Terms] OR ('patient' [All Fields] AND 'compliance' [All Fields] OR 'patient compliance' [All Fields]
OR 'compliance' [All Fields] OR 'compliance' [MeSH Terms] AND (urate-lowering [All Fields] AND ('therapy' [Subheading] OR 'therapy' [All Fields] OR 'therapeutics' [MeSH Terms] OR 'therapeutics' [All Fields]) AND ('gout' [MeSH Terms] OR 'gout' [All Fields] (see online supplementary file 1). For the Chinese databases, we used Chinese translations of terms meaning gout and adherence and ULT as free-text terms in the Chinese databases.

\section{Inclusion and exclusion criteria}

Inclusion criteria were: (1) patients with gout (defined by the American College of Rheumatology or by the articles) older than 18; (2) papers that reported adherence/ compliance data with ULT and (3) cross-sectional design or baseline cross-sectional data from a longitudinal study.

Exclusion criteria were: (1) duplicates; (2) studies on adherence to non-ULT related treatment; (3) articles on persistence, discontinuation, switching, treatment gap or 


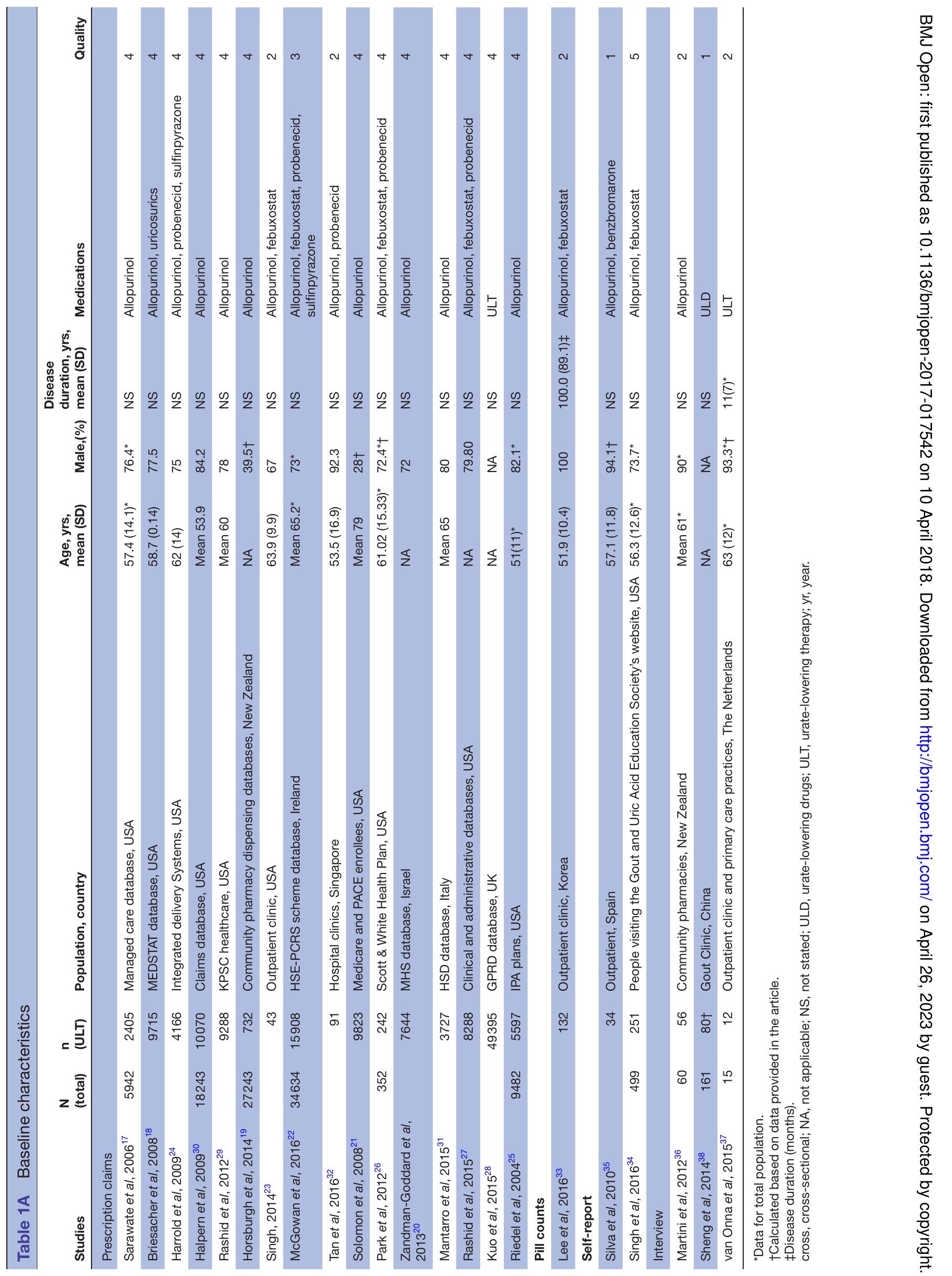




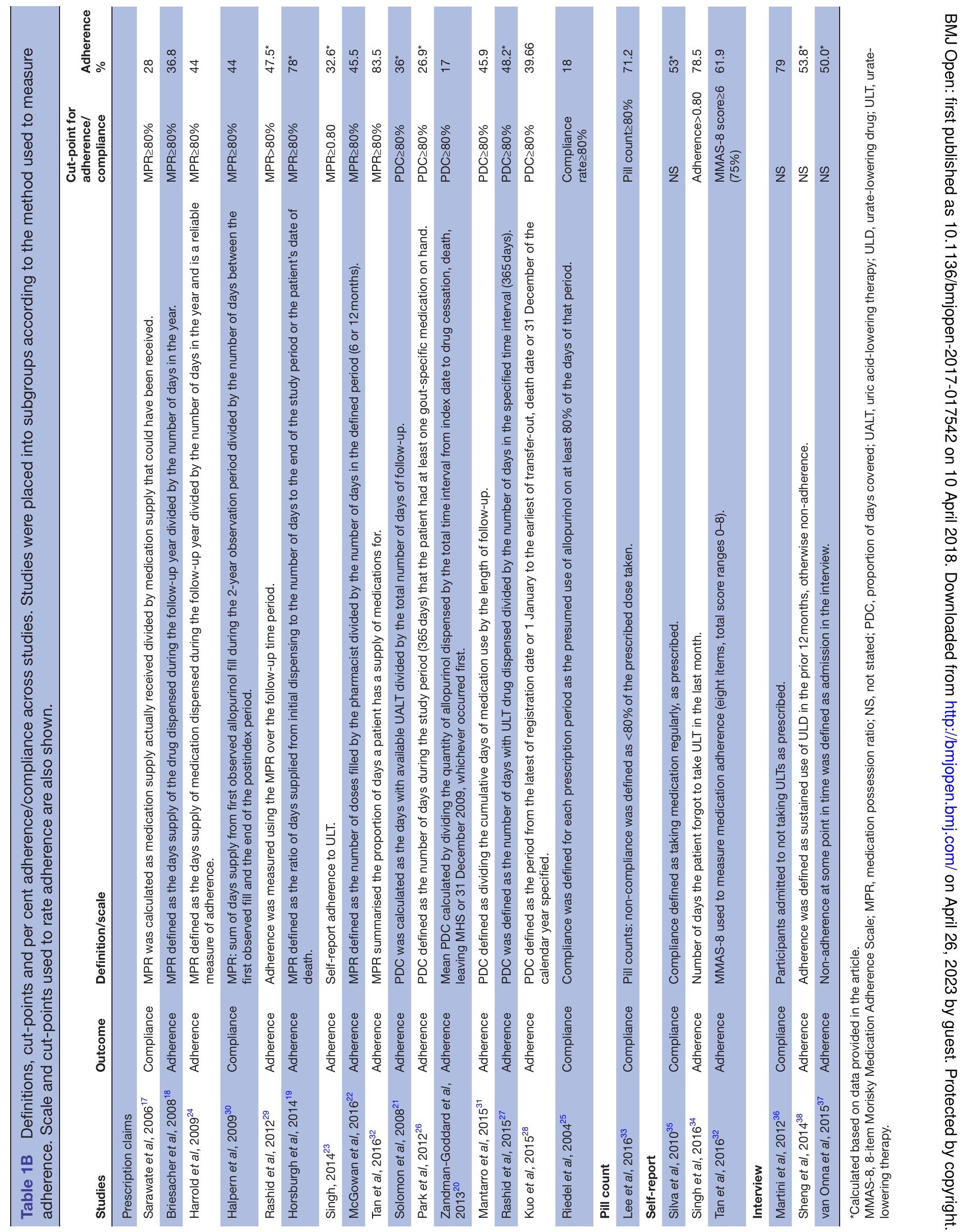




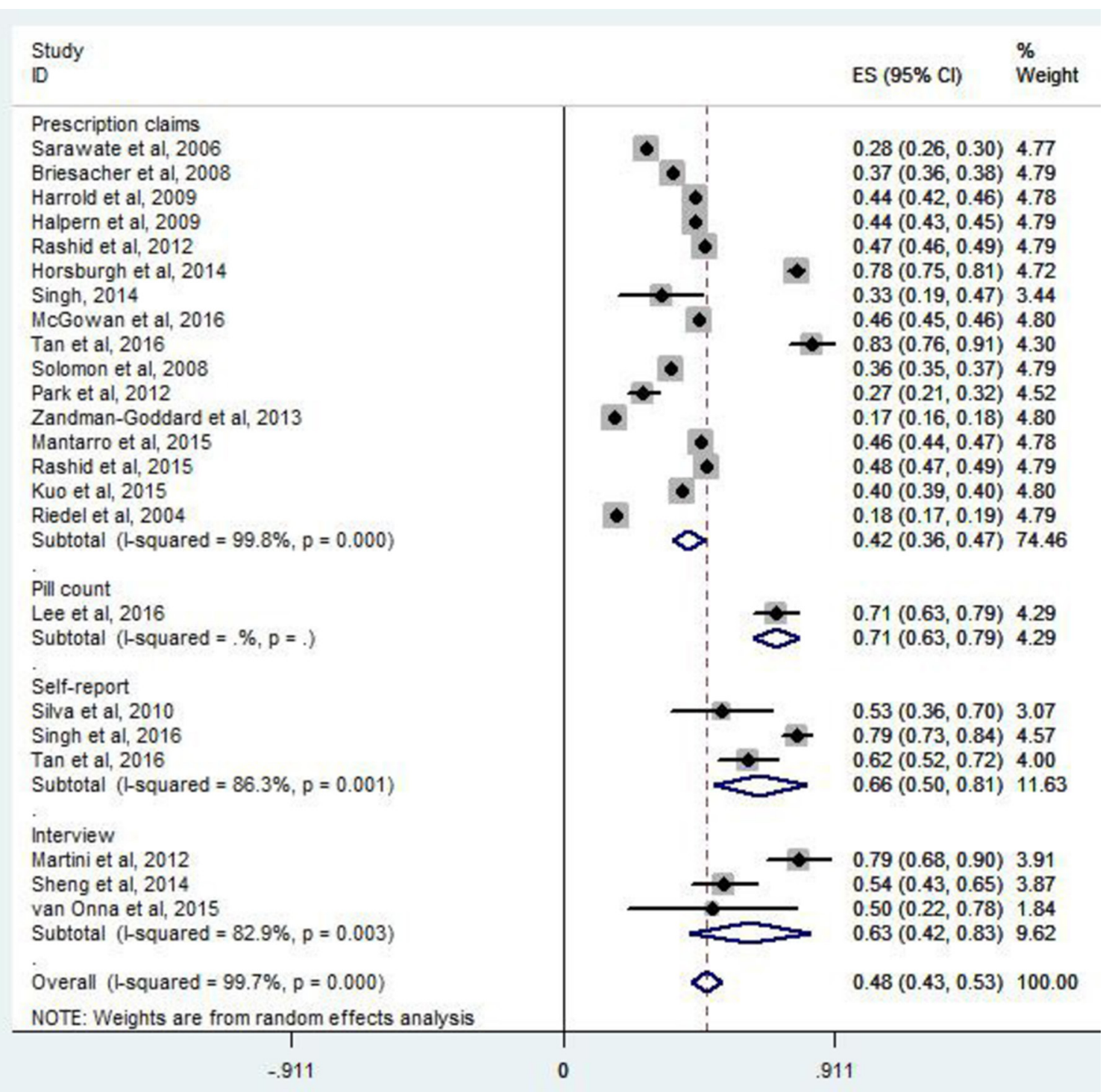

Figure 2 Meta-analysis of per cent of adherent patients by method used to measure adherence. ES, effective size.

retention rate; (4) data not independently available (eg, papers that contained data on a mix of medications, but there was no breakdown of adherence by medication) and (5) data from physicians' subject evaluation instead of objective and quantified methods.

\section{Data extraction and quality assessment}

According to the titles and abstracts, two authors (RY and LL) read the relative studies independently, and decided whether to include articles by reading the abstract and further full-text examination. Two trained investigators extracted the following information from each article independently: year, sample size, population, country, average age of participants, percentage of male participants, mean disease duration, type of medication, outcome, criteria for detection of adherence/compliance, cut-point for adherence/compliance, and reported prevalence of adherence/compliance. If we encountered multiple measurements from the same study, the most common evaluation method was used to carry out analysis. All the methods were used for subgroup analysis if not in the same subgroup. The methodological quality of each study included in the present meta-analysis was evaluated using a modified version of the NewcastleOttawa Scale, ${ }^{12}$ where studies with more than or equal to 3 points were considered having low risk of bias while those with less than 3 points were considered having high risk of bias. All discrepancies were resolved by discussion and adjudication of a third reviewer (GZ).

\section{Outcome measures}

The outcomes were adherence or compliance assessed with prescription claims (eg, medication possession ratio, proportion of days covered), pill count, self-report or interview.

\section{Statistical analysis}

We used a random-effects meta-analysis, which was preferable and can provide wider CIs, to pool studies reporting adherence rates to ULT in patients with gout. ${ }^{13} \mathrm{I}^{2}$ was used 
Table 2 Summary of adherence rate and heterogeneity findings

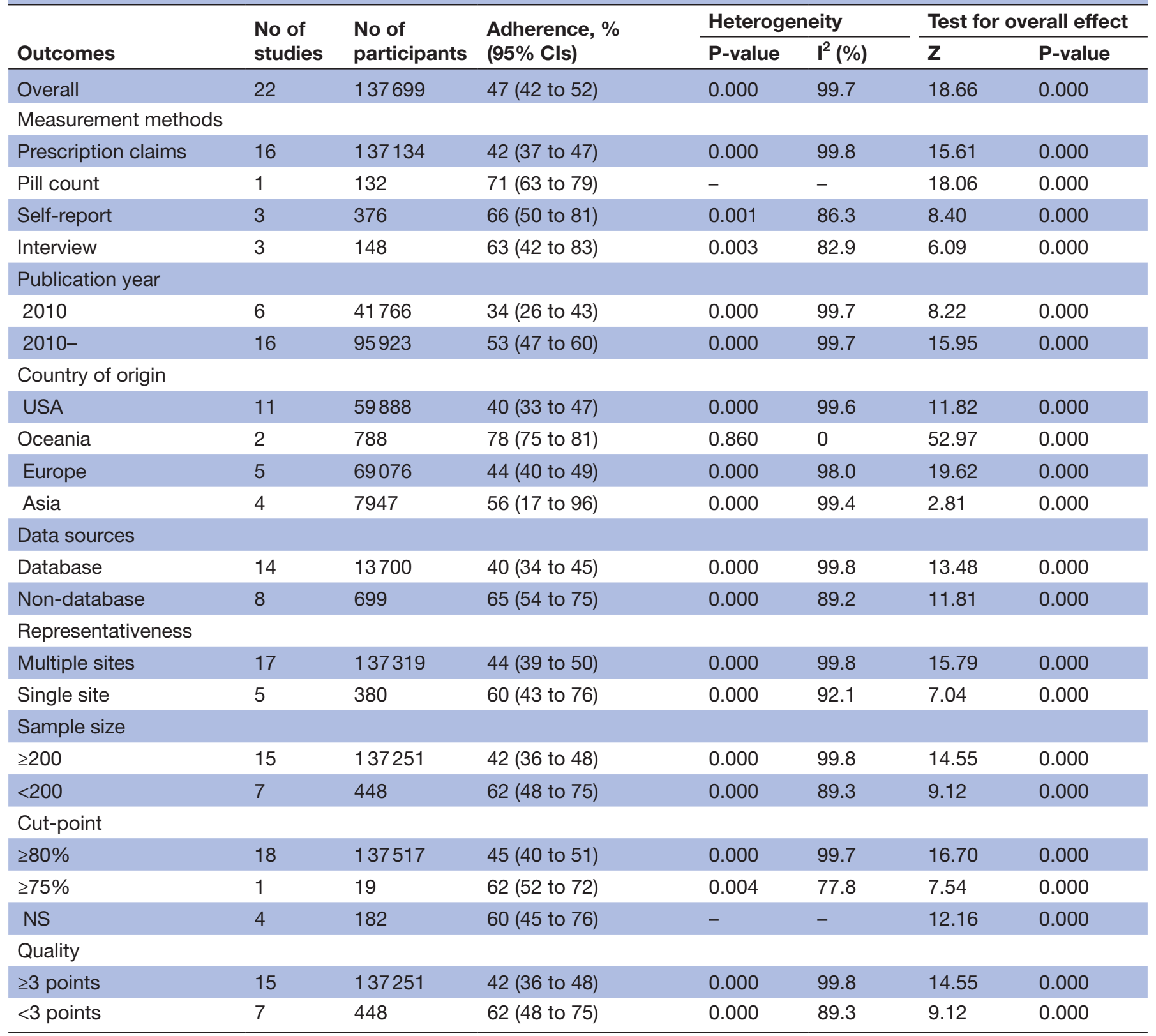

NS, not stated.

to assess between-study heterogeneity, $\mathrm{I}^{2}$ with thresholds of $\geq 25 \%$ (low heterogeneity), $\geq 50 \%$ (moderate heterogeneity) and $\geq 75 \%$ (high heterogeneity). ${ }^{14}$ A sensitivity analyses was performed for sequential omission of each study to explore individual study's impact on the overall prevalence estimate. Wherever possible, subgroup analyses were planned by measurement methods, publication year, country of origin, data sources, representativeness of the sample, sample size, cut-point and overall quality, if there was more than one study in the subgroup. We combined Funnel plots and Egger's test to explore the potential publication bias in this meta-analysis. ${ }^{1516}$ We performed regression analysis to test the difference among methods that was used to measure rate of adherence. Statistical analyses were performed with STATA V.12.0. The statistical significance level was 0.05 .

\section{RESULTS}

Study selection

After having assessed the studies by selection criteria, we included data from 22 studies, involving a total of 137699 adult patients with gout. A flow chart of the study selection process is shown in figure 1.

\section{Study characteristics}

Baseline characteristics of the included study, the methods used to evaluate adherence to ULT and the frequency of their use are presented in table $1 \mathrm{~A}$ and $\mathrm{B}$. All included 
studies assessed adherence in four different ways. Fifteen studies were assessed for adherence using prescription claims, ${ }^{17-31}$ with the cut-point of $\geq 80 \%$. One study used prescription claim and self-report, ${ }^{32}$ one article used pill count ${ }^{33}$ two used self-report ${ }^{34} 35$ and three articles were assessed by interview. ${ }^{36-38}$ Among the 22 identified studies, 11 took place in USA, 2 in Oceania, 5 in Europe and 4 in Asia. When evaluated using the Newcastle-Ottawa quality assessment criteria, out of 5 possible points, 1 study received 5 points, ${ }^{34} 13$ received 4 points, ${ }^{17-21}$ 24-31 1 received 3 points, ${ }^{22} 5$ received 2 points ${ }^{23} 32333637$ and 2 received 1 point. ${ }^{35} 38$

\section{Rate of adherence to ULT among patients with gout}

The adherence rate to ULT ranged from $17 \%$ to $83.5 \%$ in individual studies (table 1B). Overall, $47 \%$ of patients with gout were adherent to ULT (95\% CI $42 \%$ to 52\%, $\mathrm{I}^{2}=99.7 \%$ ) (figure not shown). According to prescription claims, the rate of adherence to ULT was $42 \%$ (95\% CI $37 \%$ to $\left.47 \%, \mathrm{I}^{2}=99.8 \%\right)$. The adherence rate was $71 \%$ (95\% CI 63\% to $79 \%$ ) for pill count, $66 \%$ (95\% CI $50 \%$ to $\left.81 \%, \mathrm{I}^{2}=86.3 \%\right)$ for self-report and $63 \%$ (95\% CI $42 \%$ to $83 \%, \mathrm{I}^{2}=82.9 \%$ ) for interview, respectively (figure 2 ). According to regression analysis, no significant difference was found for adherence measurement methods $(\mathrm{p}=0.535)$.

\section{Sensitivity and subgroup analyses}

Sensitivity analysis indicated that all of the estimated values were in regions of the lower CI limit and upper CI limit, which showed that no single study affected our results (figure not shown). A summary of meta-analysis and heterogeneity assessments is described in table 2 . The subgroup analysis of adherence rate to ULT estimates was conducted according to the measurement methods, publication year, country of origin, data sources, representativeness of the sample, sample size, cut-point and overall quality. The results of the meta-analysis affected by the country of origin in those included studies showed that studies from the Oceania had higher adherence estimates $(78 \%$ (95\% CI $75 \%$ to $81 \%$ ) vs $40 \%$ (95\% CI $33 \%$ to $47 \%$ ) vs $44 \%$ (95\% CI $40 \%$ to $49 \%$ ) vs $56 \%$ (95\% CI $17 \%$ to $96 \%$ ) from USA, Europe and Asia, respectively). The subgroup analysis for measurement methods, publication year, data sources, representativeness of the sample, sample size, cut-point and overall quality showed no clear patterns.

\section{Evaluation of publication bias}

No significant evidence of publication bias was found in overall analyses through the Egger's test, in any study reporting adherence according to prescription claims, self-report and interview (Egger: bias $=5.42$ (95\% CI -6.55 to 17.39$), \mathrm{p}=0.356$; Egger: bias $=4.32 \quad(95 \% \mathrm{CI}$ -16.55 to 25.18$), \mathrm{p}=0.664$; Egger: bias $=-4.92(95 \%$ CI -20.50 to 10.66$), \mathrm{p}=0.155$; Egger: bias $=-2.02$ (95\% CI -70.13 to 66.08 ), $\mathrm{p}=0.770$, respectively) (figure not shown).

\section{DISCUSSION}

To the best of our knowledge, this systematic review and meta-analysis of 22 studies involving 137699 adult patients with gout is the first to quantify adherence and to seek a relationship between adherence and the method used to measure it.

Totally, $47 \%$ adult patients with gout adhered to ULT. Majority of studies using prescription claims to report adherence to ULT were present in $42 \%$ among patients with gout (16 of 22). The rate of adherence to ULT was $71 \%, 66 \%$ and $63 \%$ for pill count, self-report and interview, respectively. The highest adherence rate measured by pill count, followed by self-report, interview and prescription claims. Although no statistical differences were found among the different methods, suboptimal medication adherence was clear across the included studies. It is particularly shocking that the adherence rate of $42 \%$ based on prescription claims and the overall adherence rate of $47 \%$ is below the well-quoted WHO estimate that $50 \%$ of adults adhere to long-term therapies.

A previous systematic review included 16 studies. ${ }^{9}$ We identified additional studies. It is important that previous reviews did not quantify adherence. In our meta-analysis, a cut-point of $\geq 80 \%$ to define adherent patients, was used in most studies. Data on persistence, discontinuation, switching, treatment gap or retention rate, as well as adherence to non-medical therapy (eg, diet recommendations) were excluded.

The results demonstrated an overall adherence rate to ULT in adult patients with gout of $47 \%$. However, heterogeneity was large. By subgroup analyses for measurement methods, publication year, country of origin, data sources, representativeness of the sample, sample size, cut-point and overall quality in those included studies, country of origin was found to have contributed to the heterogeneity between studies, with heterogeneity of $0 \%$ among studies from Oceania, 99.6\% from USA, 98.0\% from Europe and $99.4 \%$ from Asia. Although studies varied widely in terms of quality, our sensitivity analyses suggested that the adherence rate estimates were reasonably stable.

This meta-analysis indicated significant difference in adherence in claims database, especially from the USA, and also from the UK. The reasons for this could be that interview studies or postal surveys are prompting patients to self-report higher adherence. Additionally, adherence also depends on the healthcare system in which the study is done-private (with billing for drugs used) versus government funded; primary care versus secondary care, as well as severity of gout and age of patients (older patients typically will have higher adherence). This could also have an impact on the findings.

The adherence rate is surprisingly low considering that ULT does not have significant side effects or require taking tablets several times a day. It could be that patients do not think it is necessary to always take urate-lowering agents (ULAs) since they may feel asymptomatic most of the time. It could also be that ULA are not included in the medical insurance; because the price of ULA is 
higher, long-term use of ULA will cause a greater financial burden on patients with gout.

Owing to the low adherence with ULT, carrying out potential and effective interventions is vital to improve gout-related outcomes. There are some interventions that can be achieved through pharmacist-assisted or nurse-assisted programmes, that may be effective, which include initiation of prophylactic anti-inflammatory medications when starting ULT, monitoring SU regularly, frequent follow-ups and improved patient education. ${ }^{39}$ Abhishek $e t$ $a l^{40}$ and Rees $e t a l^{41}$ have confirmed that there are excellent adherence rates after nurse-led treatment of gout, which means that these interventions could improve adherence to ULT in patients with gout and, eventually, improving gout-related outcomes.

However, we still need to address additional shortcomings in this systematic review and meta-analysis. First, heterogeneity which was high among the studies remained unexplained by the variables examined. Unexamined factors, such as gender, age, disease duration and study design might contribute to the risk for adherence to ULT among patients with gout. Second, owing to lack of access, we did not include the studies from EMBASE database and Cochrane database library in our search, and several studies that referred to medications unspecified ULT were excluded, which could bias the findings.

\section{CONCLUSION}

Among adult patients with gout, overall adherence rate to ULT was as low as $47 \%$, which suggested that clinicians should pay more attention to medication adherence in patients with gout to effectively improve adherence to ULT.

Acknowledgements We thank Yuwei Gu for her great assistance with this study.

Contributors RY and LL searched and checked the databases according to the inclusion and exclusion criteria, extracted the data and assessed their quality, analysed the data and wrote the draft of the paper. GZ, YC, LZ, QZ, TF, HC, LL and ZG gave advice on meta-analysis methodology and revised the paper. All authors contributed to reviewing or revising the paper. $L L$ and $Z G$ were the guarantors of this work and had full access to all the data in the study and took responsibility for its integrity and the accuracy of the data analysis. All authors read and approved the final manuscript.

Funding This study was supported by grants from the Chinese National Natural Science Foundation (grant nos 81671616 and 81471603); Top Six Types of Talents Financial Assistance of Jiangsu Province (grant nos BRA2016527); Science Foundation of Nantong City (grant nos MS2201564 and MS22016028).

Competing interests None declared.

Patient consent Not required.

Provenance and peer review Not commissioned; externally peer reviewed.

Data sharing statement No additional data are available.

Open Access This is an Open Access article distributed in accordance with the Creative Commons Attribution Non Commercial (CC BY-NC 4.0) license, which permits others to distribute, remix, adapt, build upon this work non-commercially, and license their derivative works on different terms, provided the original work is properly cited and the use is non-commercial. See: http://creativecommons.org/ licenses/by-nc/4.0/ (c) Article author(s) (or their employer(s) unless otherwise stated in the text of the article) 2018. All rights reserved. No commercial use is permitted unless otherwise expressly granted.

\section{REFERENCES}

1. Roddy E, Doherty M. Epidemiology of gout. Arthritis Res Ther 2010;12:223.

2. Coburn BW, Bendlin KA, Sayles $H$, et al. Target serum urate: do gout patients know their goal? Arthritis Care Res 2016;68:1028-35.

3. Jordan KM, Cameron JS, Snaith M, et al. British society for rheumatology and british health professionals in rheumatology guideline for the management of gout. Rheumatology 2007;46:1372-4.

4. Zhang W, Doherty M, Bardin T, et al. EULAR evidence based recommendations for gout. Part II: management. Report of a task force of the EULAR Standing Committee for Internationa Clinical Studies Including Therapeutics (ESCISIT). Ann Rheum Dis 2006;65:1312-24.

5. Richette P, Bardin T. Gout. Lancet 2010;375:318-28.

6. Corbett EJM, Pentony P, McGill NW. Achieving serum urate targets in gout: an audit in a gout-oriented rheumatology practice. Int $J$ Rheum Dis 2017;20:894-7

7. Perez-Ruiz F, Atxotegi J, Hernando I, et al. Using serum urate levels to determine the period free of gouty symptoms after withdrawal of long-term urate-lowering therapy: a prospective study. Arthritis Rheum 2006;55:786-90.

8. World Health Organization. Adherence to long-term therapies: evidence for action. www.who.int/chp/knowledge/publications/ adherence_report/en (assessed 30 Nov 2015).

9. De Vera MA, Marcotte G, Rai S, et al. Medication adherence in gout: a systematic review. Arthritis Care Res 2014;66:1551-9.

10. Moher D, Liberati A, Tetzlaff J, et al. Preferred reporting items for systematic reviews and meta-analyses: the PRISMA statement. Ann Intern Med 2009;151:264.

11. Stroup DF, Berlin JA, Morton SC, et al. Meta-analysis of observational studies in epidemiology: a proposal for reporting. Meta-analysis Of Observational Studies in Epidemiology (MOOSE) group. JAMA 2000;283:2008-12.

12. Stang A. Critical evaluation of the Newcastle-Ottawa scale for the assessment of the quality of nonrandomized studies in metaanalyses. Eur J Epidemiol 2010;25:603-5.

13. Borenstein M, Hedges LV, Higgins JP, et al. A basic introduction to fixed-effect and random-effects models for meta-analysis. Res Synth Methods 2010;1:97-111.

14. Higgins JP, Thompson SG, Deeks JJ, et al. Measuring inconsistency in meta-analyses. BMJ 2003;327:557-60.

15. Egger M, Smith GD, Schneider M, et al. Bias in meta-analysis detected by a simple, graphical test. BMJ 1997;315:629-34.

16. Sterne JA, Egger M. Funnel plots for detecting bias in meta-analysis: guidelines on choice of axis. J Clin Epidemiol 2001;54:1046-55.

17. Sarawate CA, Brewer KK, Yang W, et al. Gout medication treatment patterns and adherence to standards of care from a managed care perspective. Mayo Clin Proc 2006;81:925-34.

18. Briesacher BA, Andrade SE, Fouayzi H, et al. Comparison of drug adherence rates among patients with seven different medical conditions. Pharmacotherapy 2008;28:437-43.

19. Horsburgh S, Norris P, Becket G, et al. Allopurinol use in a New Zealand population: prevalence and adherence. Rheumatol Int 2014;34:963-70.

20. Zandman-Goddard G, Amital H, Shamrayevsky N, et al. Rates of adherence and persistence with allopurinol therapy among gout patients in Israel. Rheumatology 2013;52:1126-31.

21. Solomon DH, Avorn J, Levin R, et al. Uric acid lowering therapy: prescribing patterns in a large cohort of older adults. Ann Rheum Dis 2008;67:609-13.

22. McGowan B, Bennett K, Silke C, et al. Adherence and persistence to urate-lowering therapies in the Irish setting. Clin Rheumatol 2016;35:715-21.

23. Singh JA. Facilitators and barriers to adherence to urate-lowering therapy in African-Americans with gout: a qualitative study. Arthritis Res Ther 2014;16:R82.

24. Harrold LR, Andrade SE, Briesacher BA, et al. Adherence with urate-lowering therapies for the treatment of gout. Arthritis Res Ther 2009;11:R46.

25. Riedel AA, Nelson M, Joseph-Ridge N, et al. Compliance with allopurinol therapy among managed care enrollees with gout: a retrospective analysis of administrative claims. J Rheumato 2004;31:1575. 
26. Park H, Rascati KL, Prasla K, et al. Evaluation of health care costs and utilization patterns for patients with gout. Clin Ther 2012;34:640-52.

27. Rashid N, Levy GD, Wu YL, YI W, et al. Patient and clinical characteristics associated with gout flares in an integrated healthcare system. Rheumatol Int 2015;35:1799-807.

28. Kuo CF, Grainge MJ, Mallen C, et al. Rising burden of gout in the UK but continuing suboptimal management: a nationwide population study. Ann Rheum Dis 2015;74:661-7.

29. Rashid N, Cheetham CT, Niu F. PMS83 adherence and uric acid goa attainment with urate lowering therapy in patients with gout. Value in Health 2012;15:A49.

30. Halpern R, Mody RR, Fuldeore MJ, et al. Impact of noncompliance with urate-lowering drug on serum urate and gout-related healthcare costs: administrative claims analysis. Curr Med Res Opin 2009;25:1711-9.

31. Mantarro S, Capogrosso-Sansone A, Tuccori M, et al. Allopurinol adherence among patients with gout: an Italian general practice database study. Int J Clin Pract 2015;69:757-65.

32. Tan C, Teng GG, Chong KJ, et al. Utility of the morisky medication adherence scale in gout: a prospective study. Patient Prefer Adherence 2016;10:2449-57.

33. Lee S, So MW, Mw S. Adherence with urate-lowering therapies among male patients with gout in a routine clinical setting. Mod Rheumatol 2016;26:950-5.
34. Singh JA, Shah N, Edwards NL. A cross-sectional internet-based patient survey of the management strategies for gout. $B M C$ Complement Altern Med 2016;16:90.

35. Silva L, Miguel ED, Peiteado D, et al. Compliance in gout patients. Acta Reumatol Port 2010;35:466-74.

36. Martini N, Bryant L, Te Karu L, KI T, et al. Living with gout in New Zealand: an exploratory study into people's knowledge about the disease and its treatment. J Clin Rheumatol 2012;18:125-9.

37. van Onna M, Hinsenveld $\mathrm{E}$, de Vries $\mathrm{H}$, et al. Health literacy in patients dealing with gout: a qualitative study. Clin Rheumato 2015;34:1599-603.

38. Sheng F, Fang W, Zeng $X$. The analysis of factors associated with treatment adherence of gout: A patient survey in china. Ann Rheum Dis 2014;732:776.

39. Aung T, Myung G, FitzGerald JD. Treatment approaches and adherence to urate-lowering therapy for patients with gout. Patient Prefer Adherence 2017;11:795-800.

40. Abhishek A, Jenkins W, La-Crette J, et al. Long-term persistence and adherence on urate-lowering treatment can be maintained in primary care-5-year follow-up of a proof-of-concept study. Rheumatology 2017;56:kew395-33.

41. Rees F, Jenkins W, Doherty M. Patients with gout adhere to curative treatment if informed appropriately: proof-of-concept observational study. Ann Rheum Dis 2013;72:826-30. 